\title{
Identification of the Alfvén Wave Resonances in the TCABR Tokamak by the Microwave Reflectometry
}

\author{
L. F. Ruchko, R. M. O. Galvão, E. A. Lerche, A. G. Elfimov, V. Bellintani Jr, \\ J. I. Elizondo, A. N. Fagundes, A. M. M. Fonseca, Yu. K. Kuznetsov, I. C. Nascimento, \\ W. P. de Sá, E. Sanada, R. P. da Silva, \\ Instituto de Física, Universidade de São Paulo, 05315-970, São Paulo, Brazil \\ and R. Valencia \\ Instituto Nacional de Investigaciones Nucleares, C.P. 11801, México, D.F., México
}

Received on 2 February, 2004; revised version received on 24 April, 2004

\begin{abstract}
The experimental results on the Alfvén mode structure identification by the microwave reflectometry in TCABR tokamak are presented. The knowledge of the spatial spectrum of the excited waves is crucial for optimization of Alfvén wave plasma heating and noninductive current drive scenarios in tokamak plasmas. No less important is the possibility to use the Alfvén wave excitation for diagnostic purposes. A microwave reflectometer with fixed frequency was used to register plasma density oscillations driven by the excited Alfvén waves, under the condition of the spectrum scanned by a controlled plasma density rise. It is shown that when the position of the local Alfvén resonance, $r_{A}$, which is defined by relation $\omega=k_{/ /}\left(r_{A}\right) C_{A}\left(r_{A}\right)$, is close to the plasma zone where the microwave signal is reflected, the high-frequency modulation of the output signal of the reflectometer at the $R F$ generator frequency increases. The possibility to use the observed effect for finding the plasma current profile in tokamaks is discussed.
\end{abstract}

\section{Introduction}

The study of the excited RF field structure is essential part of the Alfvén wave experimental program that is currently carried out in TCABR tokamak $[1,2,3]$. These experiments should help to find out the optimal conditions for antennae performance and to ensure the effective plasma heating and noninductive current drive.

In the course of these studies the spatial spectrum of the excited waves $M, N$ ( where $M$ and $N$ are poloidal and toroidal wave numbers correspondingly) is analyzed by making a comparison of signals from magnetic probes with the theoretical simulations. The shortcoming of commonly used RF magnetic probe diagnostic system, which is used for measuring the spatial spectrum and phase velocity of Alfvén waves, lies in the fact that it can pick up magnetic fields at the plasma boundary only, where plasma temperature is low. The mesurements of the Alfvén wave structure in the plasma inner regions have to be carried out by other methods. One of these methods consists of the registration of plasma density perturbations, which are driven by Alfvén waves.

In the AW heating scheme, the waves are launched by an external antenna structure. These waves, propagating towards plasma center, can be converted into kinetic Alfvén wave (KAW) at specific magnetic surfaces, where their phase velocity equals the local Alfvén velocity $C_{A}(r)=$ $B / \sqrt{\mu_{0} m_{i} n_{i}(r)}$, and are further dissipated through Landau damping on thermal electrons $\left(v_{t h} \approx v_{A}\right)$ [4]. The location of the mode conversion zone determines the RF power deposition region, where plasma density oscillations at the frequency $\omega$ of the applied RF field are excited. This position, $r=r_{A}$, depends on the RF frequency $\omega_{R F}$ and on the excited wave mode numbers $M, N$ according to the dispersion relation

$$
\omega_{R F}^{2}=k_{/ /}^{2}\left(r_{A}\right) C_{A}^{2}\left(r_{A}\right)\left(1-\omega_{R F}^{2} / \omega_{c i}^{2}\right)
$$

where

$$
k_{/ /}(r)=\frac{N}{R}\left[1+\frac{M}{N q(r)}\right]
$$

Here $B$ is the magnetic field with toroidal component $B_{T}$ and poloidal component $B_{P}, \omega_{c i}=Z e B / m_{i}$ is the ion cyclotron frequency, $m_{i}$ and $n_{i}$ are ion mass and number density, respectively, $q(r)=r B_{T} / R B_{P}(r)$ is safety factor, $R$ is the major radius of the plasma column, and $r$ is the radial coordinate of a pseudo-toroidal coordinate system centered at the magnetic axis. It is seen that the position of the $A W$ deposition zone, where intensive plasma density oscillations are excited, depends both on the plasma parameters $n(r), q(r), m_{i}$ and on the $R F$ field characteristics $M, N$, $f=\omega_{R F} / 2 \pi$.

The magnitude of plasma density oscillations in the course of the AW excitation has maximum value in the vicinity of the AW resonance layer

$$
\frac{\widetilde{n}(r)}{n}=\frac{e\left|\widetilde{E}_{/ /}\right|}{m_{e} v_{t h}^{2}\left|k_{/ /}\right|},
$$

where $\widetilde{E}_{/ /}=\sqrt{\frac{8 \sqrt{2 \pi} k_{/ /}^{3} v_{t h}^{3}}{\pi \omega_{p e}^{2} \omega_{R F}^{2}}} \widetilde{p}$ is the parallel to the mag- 
netic field component of the oscillating electric field of the $\mathrm{KAW}, v_{t h}$ is electron thermal velocity, and $\widetilde{p}(r)$ is specific dissipated $R F$ power. The numerical calculations have shown that the $R F$ input energy is deposited within few ion gyroradii of the resonance layer and density fluctuations, too, are concentrated near the resonance layer [5].

Plasma density oscillations driven by launched AW were experimentally investigated in the PRETEXT [6] and TCA [7] tokamaks. In the former device, the spatial structure of the density fluctuations associated with the excited AW was measured by laser interferometry, using a $\mathrm{CO}_{2}$-laser interferometer system that had its detection circuitry electrically referenced to the $R F$ current through a high-frequency lockin amplifier. This provided a large signal-to-noise level and insured that only driven density oscillations $\widetilde{n}$ were detected. The detected signal was a measure of the line integral of the amplitude and the phase of density fluctuations, $\widetilde{\Delta}=\left(2 \pi / \lambda n_{c}\right) \int \widetilde{n}(y) d y$, where $\lambda=10.6 \mu m$ and $n_{c} \simeq 10^{25}$ $\mathrm{m}^{-3}$ is the cutoff density. The results were in good agreement with the predictions of the kinetic theory of global Alfvén wave excitation.

In the TCA tokamak [7], the AW driven plasma density fluctuations were studied with a phase-contrast imaging interferometer [8]. This system provided a simultaneous measurement of the amplitude and phase of the line integral of the density fluctuations at sixteen different radial locations between $r / a=0$ and $r / a \cong 0.8$. These results confirmed the effect of mode conversion to the kinetic Alfvén wave, at the shear Alfvén wave resonance layers, and agreed with the KAW dispersion relation [4]. The observed wave fields were well modelled by the kinetic calculations.

These experiments $[6,7]$ have proved the possibility to drive plasma density oscillations with a predefined spatial structure, with the help of externally excited Alfvén waves, for diagnostic purposes. However, the registration and interpretation of these oscillations through the line integrated interferometer signals is rather complicated and some information about the radial structure can be inaccessible. In the sequel we present experimental results, which show that these oscillations can be detected by microwave reflectometry and an essential information about localization of RF power deposition zone and mode structure can be obtained.

Microwave reflectometry is more convenient diagnostic method for Alfvén wave detection due to its great capability to perform measurements of electron density fluctuations in tokamaks with both high temporal and spatial resolutions. A description of different techniques used in reflectometry measurements can be found in the reviews $[9,10]$. The reflectometry signals are extremely sensitive[10, 11] to plasma density oscillations in the cutoff layer. Due to this feature microwave reflectometry is now routinely used for probing the structure of magnetohydrodynamic and turbulent fluctuations in fusion plasmas.

The interpretation of reflectometer signal in the onedimensional (1-D) geometry is relatively simple. In this case, a plane stratified plasma with permittivity $\varepsilon(r)=$ $\varepsilon_{0}(r)+\delta \varepsilon(r)$, where $\varepsilon_{0}(r)$ is the permittivity of the unperturbed plasma and $\delta \varepsilon(r)=\delta n_{e} \frac{d \varepsilon_{0}}{d n_{e}}$ is fluctuating part, is probed by a wave propagating in the $r$-direction. Under these conditions the fluctuating phase of the reflected signal is given by the approximation of geometric optics[11], where $r_{c}$ is the location of the cutoff layer.

$$
\delta \phi=k_{0} \int_{0}^{r_{c}} \frac{\delta \varepsilon(r)}{\sqrt{\varepsilon_{0}(r)}} d r .
$$

For the O-mode and for a small density perturbation of the form $\delta n_{e} \cos \left[k_{r}\left(r-r_{c}\right)\right]$ simple expression for the perturbed phase $\delta \phi$ is given by

$$
\begin{array}{ll}
\delta \phi \approx 2\left(k_{0} L_{n}\right) \frac{\delta n_{e}}{n_{e}}, & k_{r} L_{n}<1, \\
\delta \phi \approx 2 k_{0}\left(\frac{L_{n}}{k_{r}}\right)^{1 / 2} \frac{\delta n_{e}}{n_{e}}, & k_{r} L_{n}>1,
\end{array}
$$

where $L_{n}$ is the density scale length at the cutoff layer, $k_{r}$ is the wave number of the density perturbation in the direction of wave propagation.[10] A general analysis of the sensitivity of reflectometry to perturbations of the plasma profile, using a full-wave description in one dimension, has proved that the approximation of geometric optics can also be applied to large perturbations also [12]. However, the limitation of the method as a linear detector of long wavelength fluctuations has to be taken into account. The application of microwave reflectometry to the study of density oscillations driven by fast magnetosonic waves in the TFTR, assuming the validity of geometric optics for reflectometer measurements, has shown that linearity to the fast wave amplitude is restricted to density fluctuation levels $\left(\delta n / n<10^{-3}\right)$ [13]. Beyond this level, over modulation of the wave front near the reflecting layer occurs what makes it difficult the interpretation of the experimental results.

The interpretation of reflectometry becomes considerably more complicated in the case of multidimensional fluctuations where, as in the case of tokamaks, the plasma permittivity varies perpendicularly to the direction of propagation of the probing wave. In this case two-dimensional full-wave simulations of reflectometry experiments are used $[14,15,16]$.

New possibilities of the diagnostic technique can be realized by integrating it with Alfvén wave (AW) excitation by external antennae. In the course of AW driving, coherent plasma density oscillations are excited in predefined plasma regions and the registration of the related perturbations by the reflectometry technique can be used for diagnostic purposes [3]. The favourable functional dependence of AW excitation zone on the plasma parameters permits easy identification of excited modes by microwave refflectometry.

In the sequel we present experimental results on the determination of these oscillations by microwave reflectometry and show how this can be used for diagnostic purposes.

\section{Theoretical considerations}

The advantage of utilization of the reflectometer technique for registration of AW driven density oscillations is clearly seen after comparison of the AW dispersion relation (Eq.1) with the expression for the reflectometer O-mode cutoff position $\omega_{p}^{2}\left(r_{c}\right)=\omega^{2}$. Since we study AW driven oscillations 
in the plasma region, where the reflectometer signal experiences cutoff, we can substitute $\omega_{p}\left(r_{A}\right)$ by reflectometer frequency $\omega$ and obtain (we have assumed here that $n_{e} \approx n_{i}$ )

$$
\frac{N}{R}\left[1+\frac{M}{N q\left(r_{A}\right)}\right] \sqrt{\frac{m_{e}}{m_{i}}}=\omega \frac{\omega_{R F}}{c \omega_{c e} \sqrt{\left(1-\omega_{R F}^{2} / \omega_{c i}^{2}\right)}}
$$

where $\omega_{c e}$ is the electron cyclotron frequency.

This equation gives the $A W$ dispersion in the plasma region where the reflectometer signal is reflected. It is seen now that all variables on the right hand side of the Eq.7 are known and hence the magnitude of the left hand side can be found. Thus, if $A W$ with predefined spectrum $M, N$ are excited in a plasma with known $m_{i}$, then the local value $q\left(r_{A}\right)$ can be found by finding the frequency, at which the reflectometer signal is modulated by $A W$ frequency, and by measuring the $r_{A}$ position by the reflectometer technique [9]. This diagnostic can be realized by sweeping the reflectometer frequency.

In the course of experiments, which were carried out in the TCABR tokamak, the reflectometer with fixed frequency $f \approx 32.3 \mathrm{GHz}$ was used, and the microwave cutoff density was given by

$$
n_{c}=\frac{\varepsilon_{0} m_{e}(2 \pi f)^{2}}{e^{2}} \approx 1.3 \times 10^{19} \mathrm{~m}^{-3}
$$

That is why in order to verify the possibility to register $A W$ driven modes by relectometer technique we have carried out the experiments with the density scan instead of reflectometer frequency sweeping. The frequency of $R F$ generator, which was used for the excitation of $A W$, has been chosen in such a way that Alfvén resonance area and the reflectometer cutoff region were overlapping in the course of a controlled density rise. In this case, the output signal of the reflectometer is modulated by the frequency of the Alfvén wave and the location of the $R F$ power deposition zone can be directly monitored. If the position of the Alfvén wave resonance zone does not coincide with the cutoff zone of the microwave reflectometer signal, then the degree of modulation of reflectometer signal by the AW frequency decreases.

The radial structure of the plasma density oscillations, which were excited by $A W$ in these experiments in TCABR tokamak, was analyzed with the help of 2-D kinetic model [17]. The calculations have shown that density oscillations are localized in the vicinity of Alfvén resonance zone. The esimates show that in this case the condition $k_{r}<k_{0} /\left(k_{0} L_{n}\right)^{1 / 3}$ is satisfied, and the approximation of geometric optics is valid[11]. The oscillating component of the signal phase, which was calculated with the help of the Eq. 4 , is equal to $\delta \phi \approx 0.3$. It means that linearity of the output signal of the reflectometer to the AW driven density oscillations is not violated.

The dependence of the Alfvén resonance position, $r_{A} / a$, for different Alfvén wave modes, and of the microwave cutoff layer, $r_{c} / a$, on the line averaged density $\left\langle n_{e}\right\rangle$ is shown in Fig. 1.

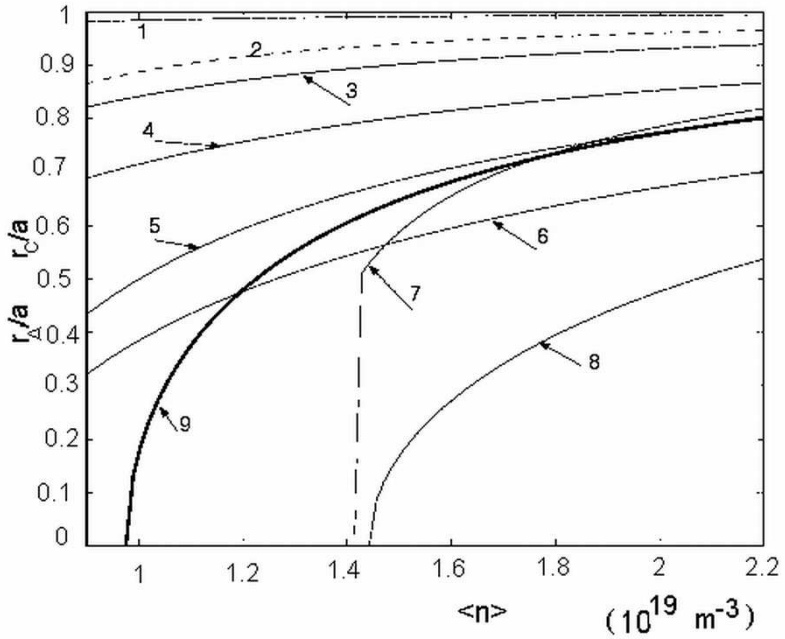

Figure 1. Dependence of the normalized Alfvén wave resonance position $r_{A} / a$ on the line averaged plasma density $\langle n\rangle$ for the modes: (1) $M= \pm 1, N=\mp 1$, (2) $M= \pm 1, N= \pm 1$, (3) $M= \pm 1, N=\mp 2$, (4) $M= \pm 2, N=\mp 3$, (5) $M=$ $\pm 1, N=\mp 3$, (6) $M= \pm 2, N=\mp 4$, (7) $M= \pm 1, N= \pm 2$, (8) $M= \pm 1, N=\mp 4$, and of the position of the reflectometer cutoff surface, $r_{c} / a(9)$.

The points for which the curves $r_{A} / a$ and $r_{c} / a$ intersect correspond to superposition of the region of the Alfvén wave resonance with that of microwave reflection, where enhancement of the reflectometer output signal is expected. The values of $r_{A}$ were calculated according to Eqs. 1 and 2, assuming that the plasma current density is given by

$$
j(r)=j(0)\left(1-\frac{r^{2}}{a^{2}}\right)^{\alpha^{j}}
$$

where $\alpha^{j}=\frac{q(a)}{q(0)}-1$, and the electron density is given by

$$
n_{e}(r)=n_{e}(0)\left(1-\frac{r^{2}}{a^{2}}\right)^{\alpha^{n}} .
$$

In these calculations the values of parameters were $\alpha^{j}=2$, $q(a)=3.2\left(I_{P}=90 \mathrm{kA}\right)$, and $\alpha^{n}=0.8$.

It is seen that, for chosen parameters, the curve, describing the position of the microwave cutoff layer, $r_{c} / a$, intersects three AW resonance zones for the mode numbers $M=$ $\pm 2, N=\mp 4$, at $<n>\simeq 1.2 \times 10^{19} \mathrm{~m}^{-3}, r_{c} / a \simeq 0.47$; $M= \pm 1, N=\mp 3$, at $\langle n\rangle \simeq 2.1 \times 10^{19} \mathrm{~m}^{-3}, r_{c} / a \simeq 0.78$; and $M= \pm 1, N= \pm 2$, at $\langle n\rangle \simeq 1.8 \times 10^{19} \mathrm{~m}^{-3}$, $r_{c} / a \simeq 0.73$. This means that only these modes can contribute to the plasma density oscillations, which can be registered by the reflectometer at the operational frequency. As consequence, these modes can be uniquely distinguished and they can be used for discharge diagnostics.

The numerical simulations have shown that TCABR antenna system can ensure sufficient spatial localization of the $R F$ power input, which permits registration of Alfvén driven density oscillations by reflectometer technique. The dependence of radial structure of $R F$ power deposition on plasma averaged density $\left\langle n_{e}\right\rangle$ is shown in Fig. 2. The calculations were carried out with the help of 1-D MHD numerical code [1]. It is seen that controlled plasma density 
scan permits to achieve the overlapping of the reflectometer cutoff zone with localized Alfvén wave driven density oscillations.

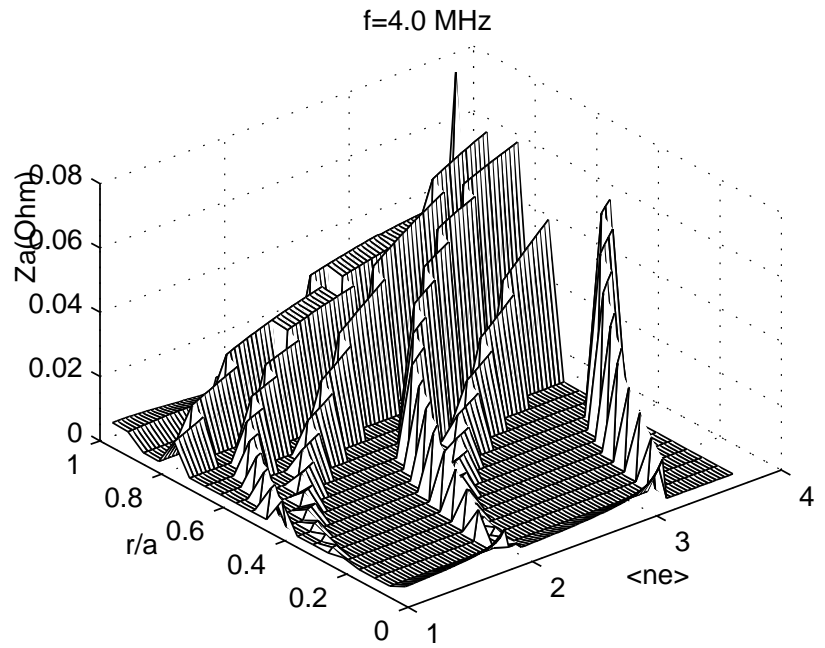

Figure 2. Dependence of the radial distribution of TCABR antenna impedance on the line averaged plasma density $\left\langle n_{e}\right\rangle$.

The phase of the density oscillations is directly related to the phase of the $R F$ antenna current and this fact permits to use the latter as reference signal in the reflectometer detection system. This allows increasing the sensitivity and improving the signal/noise ratio. The great advantage of the proposed diagnostic technique is the elimination of the influence of the reflections from the tokamak vacuum chamber elements, because only reflections from plasma cutoff layer will contribute to output signal.

\section{Experimental setup}

The experiments were carried out in the tokamak TCABR $(\mathrm{a}=0.18 \mathrm{~m}, \mathrm{R}=0.61 \mathrm{~m}, \mathrm{~B}=1.1 \mathrm{~T})[1,2]$. The basic parameters in this investigation were the following. Plasma current: $I_{P}=70$ - $95 \mathrm{kA}$; edge safety factor: $q(a) \simeq 3.1-4$; line averaged plasma density: $\langle n\rangle=(0.9-2.5) \times 10^{19} \mathrm{~m}^{-3}$; working gas: hydrogen. The $R F$ power absorbed by the plasma was limited to $\widetilde{P}_{a} \leq 40 \mathrm{~kW}$ and the frequency was fixed at $f=4.0 \mathrm{MHz}$.

The AW antenna module has two groups of RF current carrying straps. These groups are positioned in two toroidal cross-sections, which are separated toroidally by an angle $\simeq 20^{\circ}$. Each group consists of two straps that are positioned symmetrically in relation to the equatorial plane of the vacuum chamber at the poloidal coordinates $\pm 45^{\circ}$. The poloidal extension of each strap is approximately $90^{\circ}$. In the basic regime of operation, the straps in the same toroidal crosssections are fed by RF currents with $(0, \pi)$ phasing so that they can excite mainly the modes $M= \pm 1, N= \pm 1, \pm 2 \ldots$

The AW antennae have no Faraday screens. Instead lateral protection is provided by boron nitride $\mathrm{BN}$ side plates, fixed directly on both sides of each strap, thus avoiding additional supports and ensuring individual protection from ion and electron toroidal fluxes.
The diagnostic tools include conventional electrotechnical measurements of the loop voltage $U_{L}$, plasma current $I_{P}$, plasma column shift $(\Delta R, \Delta z)$, plasma energy content, by the diamagnetic signal and calculated plasma equilibrium parameters, optical measurements of the $H_{\alpha}$ radiation, and fast bolometer measurements. The line averaged plasma density was measured by a three-channel microwave interferometer.

A schematic representation of the reflectometer diagnostic scheme is shown in Fig. 3. The reflectometer operates in the continuous regime at the fixed frequency $f \approx 32.3$ $\mathrm{GHz}$, which corresponds to a cutoff plasma density $n_{c} \approx$ $1.3 \times 10^{19} \mathrm{~m}^{-3}$. The microwave power is fed from the klystron oscillator (1) to the antenna ( 8 ) by a oversized waveguide. The same antenna (8) is used as transmitter and receiver. It consists of a circular horn terminated by a lens. The antenna is installed in the tokamak midplane in the immediate vicinity of the glass window of the tokamak vacuum chamber. The antenna spot is on the order of $4-6 \mathrm{~cm}$ in diameter over the full range of reflection layer locations $\left(r_{c} \approx 5-15 \mathrm{~cm}\right)$, which were used in these experiments, and it is significanly smaller than transverse perturbation wavelengths for modes $M= \pm 1, N= \pm 1, \pm 2$.

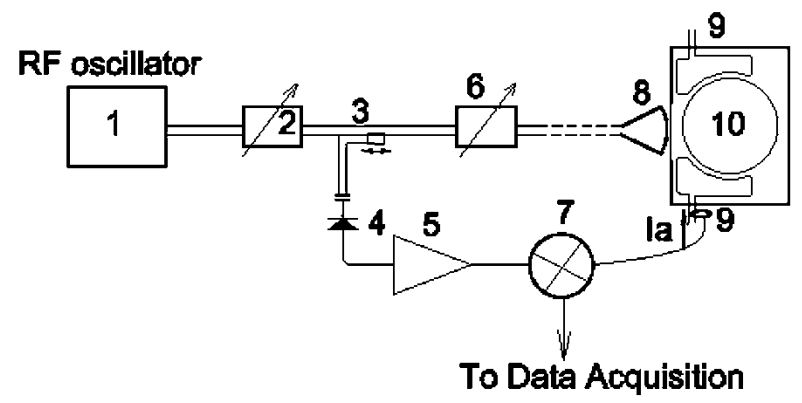

Figure 3. Schematic representation of the reflectometer diagnostic scheme: 1- microwave klystron oscillator, $f=32.3 \mathrm{GHz}, 2$ - attenuator; 3 - directional coupler; 4 - diode detector; 5 - resonance amplifier; 6 - phase shifter; 7 - analog multiplier; 8 - horn antenna; 9 - $R F$ Alfvén antennae, 10 - plasma column.

The output signal is amplified by the selective amplifier (5) with passband frequency $f=(4.0 \pm 0.1) \mathrm{MHz}$. Then the signal is multiplied in the analog multiplier (7) by the reference signal, which is proportional to $R F$ antenna (9) current, and is filtered by the low-pass filter with pass band $f \leq 100 \mathrm{kHz}$. The analog multiplier operates in a regime, for which the output signal does not depend on the amplitude of the reference signal, but only on the amplitude of the reflectometer signal and on the phase shift between them. In this way only $R F$ modulated microwave signals, which are caused by the $R F$ driven density oscillations, are registered by the data acquisition system of TCABR. The advantage of this technique is the elimination of the influence of the reflections from the tokamak vacuum chamber elements, because only $R F$ modulated reflections from plasma cutoff layer contribute to output reflectometer signal.

The data analysis includes the determination of the amplitude and spectral characteristics of these signals and their dependence on the variation of line averaged plasma density $<n(t)>$. 
SHOT \#10677
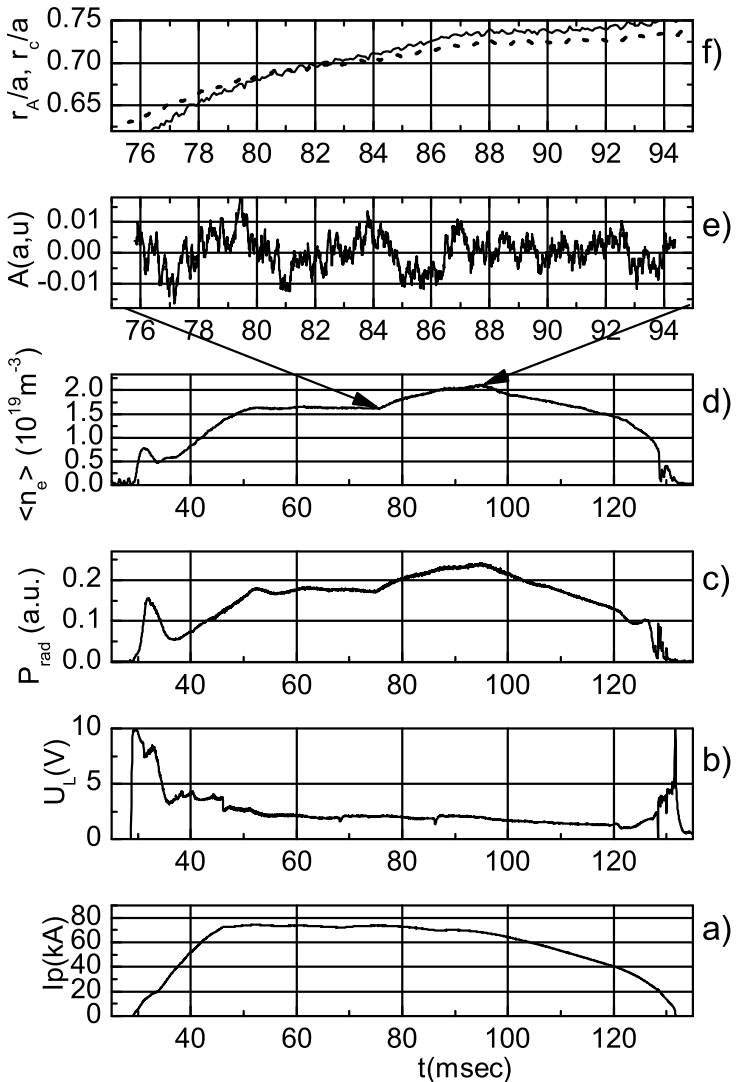

Figure 4. Shot \#10677: a) - the plasma current $I_{P}$; b) - loop voltage $U_{L} ;$ c) - radiated power $P_{\text {rad }} ;$ d) - line averaged plasma density $\left\langle n_{e}\right\rangle$; e) - the time trace of the expanded reflectometer output signal $\widetilde{A}$ during $R F$ pulse; f) - the radial resonance position $r_{A} / a$ (solid line) for mode numbers $M= \pm 1, N= \pm 2$, and the reflectometer cutoff position $r_{c} / a$ (dotted line).

\section{Results}

The registration of Alfvén wave resonances by microwave reflectometry was studied in the typical experimental conditions of the tokamak TCABR. The initial rather low value of the line averaged plasma density, $\langle n\rangle \approx(1.0-1.6) \times 10^{19}$ $\mathrm{m}^{-3}$, was chosen in order to ensure that the positioning of the microwave cutoff layer $n_{c} \approx 1.3 \times 10^{19} \mathrm{~m}^{-3}$ was in the inner plasma region. In the course of the experiments, both the initial plasma density, before application of the $R F$ pulse, and the rate of plasma density increase, by fast gas puffing during Alfvén wave pulse, were varied and the corresponding variations in the reflectometer signal were determined. At the time $t=75 \mathrm{msec}$, when the plasma current $I_{p}$ reached its maximum value and the plasma parameters were in the quasistationary phase, the $R F$ generator was triggered on with a pulse duration $\tau_{A} \approx 20 \mathrm{msec}$. Simultaneously the controlled pulsed gas puffing was initiated. The temporal variation of the fast gas puffing was adjusted such that plasma density increased during the $R F$ pulse and therefore the zones of both Alfvén resonance and microwave cutoff were swept. The time traces of some basic discharge parameters are shown in Fig. 4 and Fig. 5 for two consecutive TCABR plasma discharges with different base plasma density.

SHOT \#10678
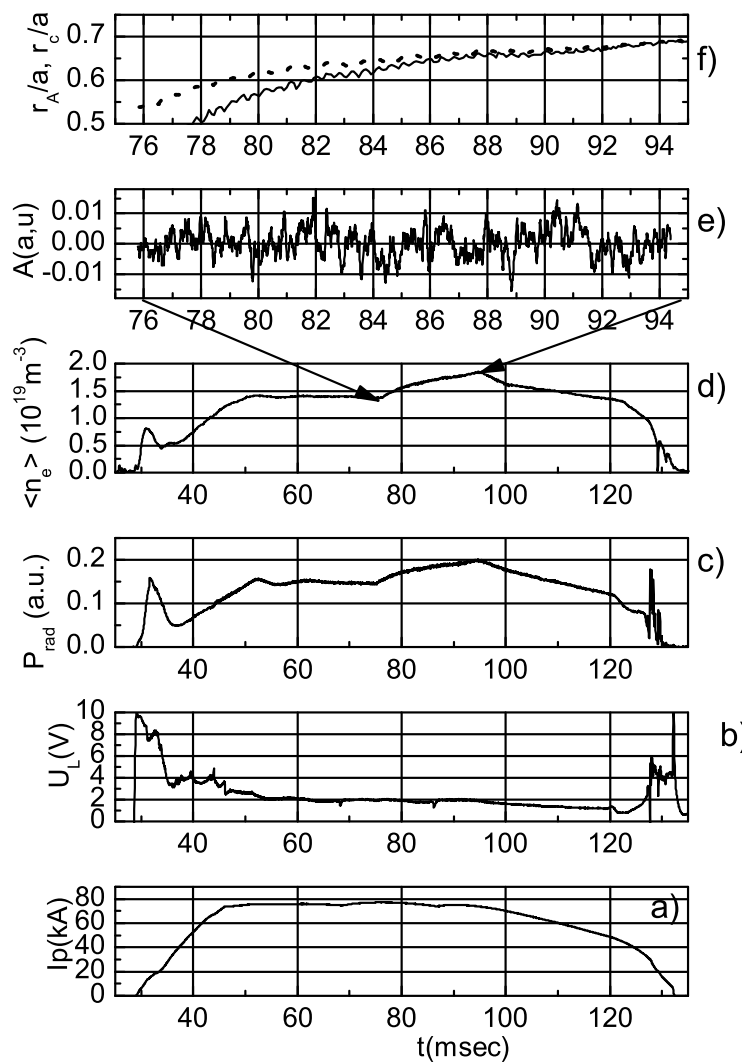

Figure 5. Shot \#10678: a) - the plasma current $I_{P}$; b) - loop voltage $U_{L} ;$ c) - radiated power $P_{r a d} ;$ d) - line averaged plasma density $<n_{e}>$; e) - the time trace of the expanded reflectometer output signal $\widetilde{A}$ during $R F$ pulse; f) - the radial resonance position $r_{A} / a$ (solid line) for mode numbers $M= \pm 1, N= \pm 2$, and the reflectometer cutoff position $r_{c} / a$ (dotted line).

During the $R F$ pulse, the level of noise increases substantially in all channels of the microwave interferometer. Fortunately, for our experimental conditions, we found that the bolometer signal is approximately proportional to the plasma density and immune to RF noise, so that it can be used to help on eliminating the noise fluctuations of the density measurements. The resulting density time traces (plot $d$ ) can be seen in Fig. 4 and Fig. 5, together with plasma current (plot $a$ ), loop voltage (plot $b$ ), and the bolometer (plot $c$ ) signals, for reference. The expanded reflectometer signals during the $R F$ pulse are shown in Fig.4(e) and Fig.5(e). The corresponding time variation of the spectrum of the reflectometer output signal $\widetilde{A}$ is shown in Fig. 6 and Fig. 7. The spectrum was determined by a moving fast Fourier transform of the reflectometer data. 
It can be seen that the characteristics the reflectometer output signal are quite different for these two shots with similar parameters. There are strong low frequency oscillations at the initial phase of the $R F$ pulse in the shot \#10677 (Fig.4(f)), which decrease to the end of the $R F$ pules. On the contrary, in the shot \#10678 low frequency oscillations of the the reflectometer output signal have begun to grow in the final stage of the $R F$ pulse.

In order to identify the origin of these spectrum changes, an analysis of the $R F$ driven Alfvén modes, whose localization can coincide with the microwave cutoff zone, has been carried out. Because the Alfvén wave resonance condition depends both on the safety factor $q(r)$ and plasma density $n(r)$ profiles (Eqs.1, 2), the analysis was carried out for different values of the profile indexes $\alpha^{j}$ and $\alpha^{n}$, keeping constraints imposed by the measured values of the plasma current $I_{p}(t)$ and line averaged density $\left\langle n_{e}\right\rangle$. The results of calculations are shown in Fig.4(f) and Fig.5(f). Here the calculated value of the radial resonance position $r_{A} / a$ for mode numbers $M= \pm 1, N= \pm 2$ where Alfvén wave driven density oscillations are localized, is plotted by solid line. The position of reflectometer cutoff zone $r_{c} / a$ is plotted by dotted line.

It is seen that due to the higher base plasma density in the shot \#10677 the zone of Alfvén wave driven density oscillations has begun to overlap the reflectometer cutoff zone at the initial phase of the $R F$ pulse, and it manifested itself by strong low frequency oscillations of reflectometer output signal. In the shot \#10678 the base plasme density was lower and this effect occured at the final stage of the $R F$ pulse.

These experimental data confirm the theoretical predictions and demonstrate that the temporal behaviour of the output signal of the reflectometer, correlated with the RF modulation of plasma density oscillations, depends strongly on the evolution of the plasma density. The amplitude of low frequency part of the registered signal increased substantially when the position of the Alfvén resonance crossed the microwave cut-off zone.

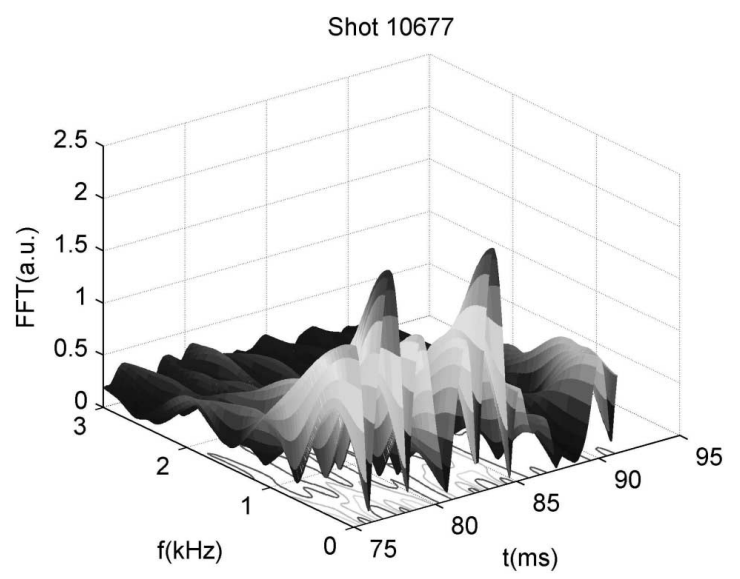

Figure 6. Shot \#10677: The time variation of the spectrum of the reflectometer output signal.

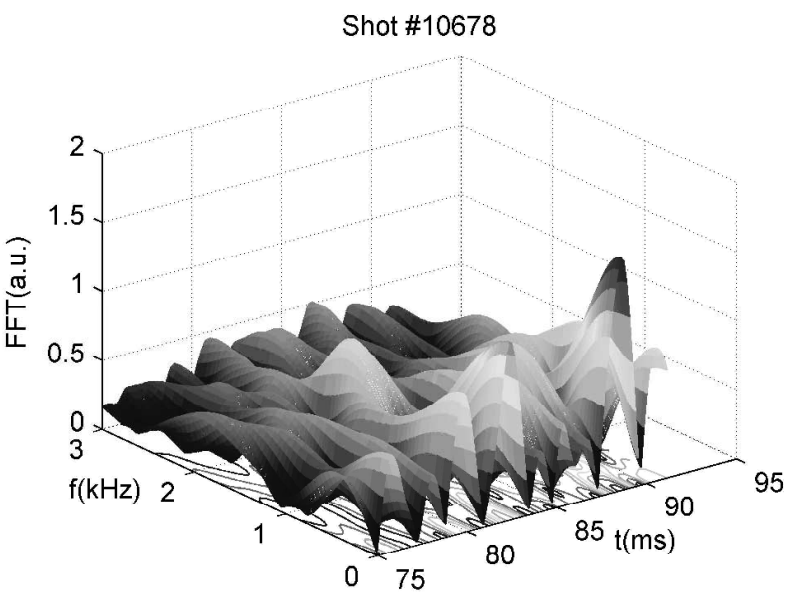

Figure 7. Shot \#10678: The time variation of the spectrum of the reflectometer output signal.

The analysis of the experimental data can also provide very important information about the effective mass of the plasma ions. In fact, the Alfvén velocity depends on the ion effective mass, $C_{A}(r)=B / \sqrt{\mu_{0} m_{i}^{e f f} n_{i}(r)}$, and results of calculations demonstrate that $r_{A} \times r_{c}$ crossing depends rather sensitively on $m_{i}^{e f f}$.

\section{Conclusions}

A microwave reflectometry scheme to detect density fluctuations correlated with the excitation of externally driven Alfvén waves has been described and the experimental results presented. In conjuction with other diagnostic methods, this method can give information about the localization of the $R F$ power deposition zone in Alfvén wave plasma heating and current drive experiments. It also allows finding the plasma current profile in the region of the RF power deposition and the effective mass of the plasma ions. The advantage of this technique is the elimination of the influence of the reflections from the tokamak vacuum chamber elements, because only reflections from plasma cutoff layer contribute to output reflectometer signal.

The experiments, which were carried out in the TCABR, are not yet complete and have to be considered as a preliminary proof of principle. The reflectometer technique and the system for Alfvén mode identification are currently being improved to provide more clear results.

\section{Acknowledgments}

This work has been supported by The National Council for Scientific and Technological Development, The State of São Paulo Research Foundation, and The International Collaboration Council of University of São Paulo.

\section{References}

[1] L. F. Ruchko, E. Ozono, R. M. O. Galvão, I.C. Nascimento, F.T. Degasperi, and E. Lerche, Fusion Engineering and Design, 43, 15 (1998). 
[2] L. F. Ruchko, E. Lerche, R. M. O. Galvão, A. G. Elfimov, I. C. Nascimento, W. P. de Sá, E. Sanada, J. I. Elizondo, A. A. Ferreira, S. Saettone, J. H. F. Severo, V. Bellintani, O. C. Usuriaga, Braz. J. Phys. 32, 57 (2002).

[3] L. F. Ruchko, R. Valencia, R. M. O. Galvão, E. A. Lerche, A. G. Elfimov, V. Bellintani Jr, J. I. Elizondo, A. N. Fagundes, A. M. M. Fonseca, Yu.K. Kuznetsov, I. C. Nascimento, W. P. de Sá, E. Sanada, and R. P.da Silva, Rev. Sci. Instrum. 75, 655 (2004).

[4] A. Hasegava and L. Chen, Phys. Fluids 19, 1924 (1976).

[5] D. W. Ross, G. L. Chen, and S. M. Mahajan, Phys. Fluids 25, 652 (1982).

[6] T. E. Evans, P. M. Valanju, J. F. Benesch, Roger D. Bengtson, Y. -M. Li, S. M. Mahajan, M. E. Oakes, D. W. Ross, X.-Z. Wang, and J. G. Watkins, Phys. Rev. Lett. 53, 1743 (1984).

[7] H. Weisen, K. Appert, G. G. Borg, B. Joye, A. J. Knight, J. B. Lister, and J. Vaclavik, Phys. Rev. Lett. 63, 2476 (1989).

[8] H. Weisen, Rev. Sci. Instrum. 59, 1544 (1988).

[9] C. Laviron, A. J. H. Donné, M. E. Manso, and J. Sanchez, Plasma Phys. Control. Fusion 38, 905 (1996).
[10] R. Nazikian, G. J. Kramer, and E. Valeo, Phys. Plasmas 5, 1840 (2001).

[11] E. Mazzucato, Rev. Sci. Instrum. 69, 2201 (1998).

[12] I. H. Hutchinson, Plasma Phys. Controllcd Fusion 34, 1225 (1992).

[13] R. Nazikian, R. Majeski, E. Mazzucato, M. McCarthy, H. K. Park, C. K. Phillips,J. H. Rogers, G. Schilling, and G. Taylor, Rev. Sci. Instrum. 68, 450 (1997).

[14] L. G. Bruskin, N. Oyama, K. Shinohara, Y. Miura, Y. Kogi, A. Mase, M. Hasegawa, and K. Hanada, Rev. Sci. Instrum. 74, 1473 (2203).

[15] S. Heuraux, S. Hacquin, F. da Silva, F. Clairet, R. Sabot, and G. Leclert, Rev. Sci. Instrum. 74, 1501 (2003).

[16] G. J. Kramer, R. Nazikian, and E. Valeo, Rev. Sci. Instrum. 74, 1421 (2003).

[17] G. Amarante-Segundo, A.G. Elfimov, D.W. Ross, R.M.O. Galvao, and I.C. Nascimento, Phys. Plas. 6, 2437 (1999). 